Supporting Information, table S1.

\title{
Abundance of species
}

\begin{tabular}{|c|c|c|c|c|c|c|c|c|c|}
\hline Species & Labels & P1 & P6 & P11 & P13 & P14 & P15 & Total & Ind_ha \\
\hline Ambelania_acida & Ambaci & 3 & 4 & 12 & 13 & 9 & 18 & 59 & 1,57 \\
\hline Anacardium_spruceanum & Anaspr & 10 & 2 & 8 & 4 & 9 & 9 & 42 & 1,12 \\
\hline Andira_coriacea & Andcor & 6 & 7 & 6 & 3 & 7 & 10 & 39 & 1,04 \\
\hline Bocoa_prouacensis & Bocpro & 78 & 47 & 91 & 100 & 86 & 80 & 482 & 12,85 \\
\hline Brosimum_guianense & Brogui & 1 & 16 & 11 & 8 & 13 & 16 & 65 & 1,73 \\
\hline Brosimum_rubescens & Brorub & 6 & 10 & 7 & 12 & 5 & 12 & 52 & 1,39 \\
\hline Carapa_surinamensis & Carsur & 34 & 36 & 47 & 31 & 28 & 32 & 208 & 5,55 \\
\hline Caryocar_glabrum & Cargla & 9 & 9 & 12 & 4 & 11 & 7 & 52 & 1,39 \\
\hline Catostemma_fragrans & Catfra & 15 & 16 & 41 & 24 & 23 & 34 & 153 & 4,08 \\
\hline Chaetocarpus_schomburgkianus & Chasch & 13 & 28 & 40 & 12 & 36 & 32 & 161 & 4,29 \\
\hline Chaetocarpus_sp.1 & Chasp1 & 7 & 8 & 21 & 6 & 6 & 5 & 53 & 1,41 \\
\hline Chaunochiton_kappleri & Chakap & 8 & 6 & 13 & 13 & 2 & 7 & 49 & 1,31 \\
\hline Chrysophyllum_prieurii & Chrpri & 18 & 15 & 32 & 9 & 20 & 18 & 112 & 2,99 \\
\hline Chrysophyllum_sanguinolentum & Chrsan & 6 & 8 & 17 & 7 & 29 & 16 & 83 & 2,21 \\
\hline Conceveiba_guianensis & Congui & 17 & 22 & 7 & 18 & 1 & 13 & 78 & 2,08 \\
\hline Couepia_bracteosa & Coubra & 8 & 12 & 14 & 6 & 10 & 3 & 53 & 1,41 \\
\hline Couepia_guianensis & Cougui & 6 & 3 & 13 & 7 & 3 & 10 & 42 & 1,12 \\
\hline Couratari_multiflora & Coumul & 28 & 33 & 67 & 29 & 27 & 23 & 207 & 5,52 \\
\hline Dicorynia_guianensis & Dicgui & 33 & 42 & 56 & 35 & 22 & 37 & 225 & 6 \\
\hline Drypetes_variabilis & Dryvar & 6 & 5 & 11 & 16 & 17 & 14 & 69 & 1,84 \\
\hline Duguetia_calycina & Dugcal & 6 & 4 & 16 & 5 & 19 & 17 & 67 & 1,79 \\
\hline Duroia_longiflora & Durlon & 8 & 8 & 8 & 4 & 15 & 7 & 50 & 1,33 \\
\hline Eperua_grandiflora & Epegra & 22 & 66 & 162 & 65 & 41 & 33 & 389 & 10,37 \\
\hline Eschweilera_congestiflora & Esccon & 9 & 15 & 29 & 15 & 6 & 22 & 96 & 2,56 \\
\hline Eschweilera_coriacea & Esccor & 62 & 36 & 9 & 23 & 40 & 8 & 178 & 4,75 \\
\hline Eschweilera_sagotiana & Escsag & 221 & 151 & 276 & 255 & 266 & 308 & 1477 & 39,39 \\
\hline Garcinia_benthamiana & Garben & 14 & 1 & 4 & 25 & 5 & 26 & 75 & 2 \\
\hline Garcinia_madruno & Garmad & 1 & 2 & 4 & 6 & 8 & 21 & 42 & 1,12 \\
\hline Goupia_glabra & Gougla & 17 & 18 & 18 & 13 & 22 & 11 & 99 & 2,64 \\
\hline Gustavia_hexapetala & Gushex & 43 & 38 & 48 & 34 & 37 & 72 & 272 & 7,25 \\
\hline Hebepetalum_humiriifolium & Hebhum & 8 & 11 & 36 & 16 & 9 & 23 & 103 & 2,75 \\
\hline Hevea_guianensis & Hevgui & 9 & 24 & 16 & 15 & 9 & 9 & 82 & 2,19 \\
\hline Hirtella_bicornis & Hirbic & 21 & 8 & 23 & 12 & 5 & 13 & 82 & 2,19 \\
\hline Inga_loubryana & Inglou & 12 & 8 & 23 & 8 & 16 & 12 & 79 & 2,11 \\
\hline Iryanthera_hostmannii & Iryhos & 59 & 21 & 17 & 87 & 19 & 24 & 227 & 6,05 \\
\hline Iryanthera_sagotiana & Irysag & 49 & 27 & 24 & 32 & 50 & 30 & 212 & 5,65 \\
\hline Jacaranda_copaia & Jaccop & 13 & 15 & 15 & 11 & 7 & 9 & 70 & 1,87 \\
\hline Lacmellea_aculeata & Lacacu & 4 & 4 & 8 & 10 & 7 & 9 & 42 & 1,12 \\
\hline Lecythis_persistens & Lecper & 290 & 202 & 258 & 243 & 259 & 222 & 1474 & 39,31 \\
\hline
\end{tabular}




\begin{tabular}{|c|c|c|c|c|c|c|c|c|c|}
\hline ecythis_poiteaui & Lecpoi & 14 & 11 & 30 & 13 & 18 & 22 & 108 & 2,88 \\
\hline Licania_alba & Licalb & 210 & 112 & 201 & 192 & 178 & 131 & 1024 & 27,31 \\
\hline cania_canescens & Liccan & 42 & 47 & 3 & 31 & 50 & 27 & 200 & 5,33 \\
\hline icania_heteromorpha & Lichet & 76 & 26 & 117 & 61 & 93 & 150 & 523 & 13,95 \\
\hline cania_membranacea & Licmem & 75 & 30 & 80 & 71 & 38 & 37 & 331 & 8,83 \\
\hline icania_micrantha & Licmic & 23 & 9 & 30 & 47 & 20 & 30 & 159 & 4,24 \\
\hline cania_ovalifolia & Licova & 13 & 11 & 12 & 12 & 14 & 21 & 83 & 2,21 \\
\hline cania_sprucei & Licspr & 10 & 11 & 16 & 17 & 18 & 31 & 103 & 2,75 \\
\hline ueheopsis_rugosa & Luerug & 9 & 1 & 2 & 10 & 8 & 10 & 40 & 1,07 \\
\hline labea_piriri & Mabpir & 11 & 11 & 1 & 17 & 13 & 7 & 60 & 1,6 \\
\hline Manilkara_bidentata & Manbid & 4 & 2 & 19 & 9 & 7 & 7 & 48 & 1,28 \\
\hline laytenus_oblongata & Mayobl & 14 & 10 & 14 & 12 & 3 & 18 & 71 & 1,89 \\
\hline liconia_tschudyoides & Mictsc & 3 & 7 & 17 & 1 & 17 & 4 & 49 & 1,31 \\
\hline Micropholis_egensis & Micege & 4 & 9 & 6 & 5 & 9 & 16 & 49 & 1,31 \\
\hline nicropholis_guyanensis & Micguy & 10 & 4 & 7 & 18 & 12 & 11 & 62 & 1,65 \\
\hline 1ouriri_crassifolia & Moucra & 12 & 15 & 36 & 25 & 32 & 18 & 138 & 3,68 \\
\hline ia_coutinhoi & Ormcou & 15 & 6 & 6 & 25 & 9 & 6 & 67 & 1,79 \\
\hline xandra_asbeckii & Oxaasb & 75 & 116 & 165 & 87 & 97 & 125 & 665 & 17,73 \\
\hline campestris & Parcam & 8 & 4 & 16 & 16 & 5 & 4 & 53 & 1,41 \\
\hline nsi & lains & 9 & 1 & 14 & 5 & 4 & 15 & 48 & 1,28 \\
\hline ogonophora_sch & Pogsch & 70 & 120 & 173 & 78 & 67 & 135 & 643 & 17,15 \\
\hline ba_g & Porgui & 19 & 10 & 7 & 9 & 27 & 15 & 87 & 2,32 \\
\hline osoqueria_latifolia & Poslat & 9 & 6 & 45 & 7 & 18 & 8 & 93 & 2,48 \\
\hline _ambelaniifolia & Pouamb & 5 & 7 & 11 & 4 & 8 & 11 & 46 & 1,23 \\
\hline _eugeniifolia & Poueug & 7 & 7 & 10 & 7 & 5 & 23 & 59 & 1,57 \\
\hline _gongrijpii & Pougon & 26 & 7 & 9 & 2 & 14 & 7 & 65 & 1,73 \\
\hline guiar & Pougui & 21 & 3 & 11 & 6 & 7 & 20 & 68 & 1,81 \\
\hline torta & Poutor & 10 & 3 & 19 & 3 & 12 & 15 & 62 & 1,65 \\
\hline cochle & Pracoc & 20 & 55 & 101 & 23 & 58 & 29 & 286 & 7,63 \\
\hline opacum & Proopa & 36 & 20 & 28 & 41 & 21 & 7 & 153 & 4,08 \\
\hline ubse & Prosub & 8 & 7 & 3 & 12 & 9 & 9 & 48 & 1,28 \\
\hline rosea & Quaros & 76 & 1 & 83 & 23 & 5 & 1 & 189 & 5,04 \\
\hline xylon_spe & Recspe & 27 & 30 & 31 & 44 & 34 & 42 & 208 & 5,55 \\
\hline acoglottis_guianensis & Sacgui & 12 & 7 & 9 & 7 & 5 & 9 & 49 & 1,31 \\
\hline _rubra & Sexrub & 20 & 10 & 10 & 9 & 14 & 17 & 80 & 2,13 \\
\hline imaba_cedron & Simced & 53 & 27 & 40 & 34 & 33 & 31 & 218 & 5,81 \\
\hline terculia_pruriens & Stepru & 14 & 20 & 17 & 26 & 19 & 23 & 119 & 3,17 \\
\hline lia_speciosa & Stespe & 8 & 14 & 9 & 2 & 4 & 7 & 44 & 1,17 \\
\hline wartzia_guianensis & Swagui & 9 & 8 & 16 & 18 & 3 & 15 & 69 & 1,84 \\
\hline Swartzia_polyphylla & Swapol & 6 & 11 & 11 & 16 & 13 & 16 & 73 & 1,95 \\
\hline Symphonia_globulifera & Symglo & 24 & 5 & 6 & 27 & 10 & 11 & 83 & 2,21 \\
\hline Symphonia_sp.1 & Symsp1 & 58 & 75 & 47 & 54 & 83 & 80 & 397 & 10,59 \\
\hline Tachigali_melinonii & Tacmel & 6 & 9 & 6 & 3 & 11 & 4 & 39 & 1,04 \\
\hline Talisia_hexaphylla & Talhex & 10 & 10 & 13 & 11 & 5 & 16 & 65 & 1,73 \\
\hline Talisia_praealta & Talpra & 6 & 5 & 8 & 8 & 4 & 8 & 39 & 1,04 \\
\hline Talisia_simaboide & Talsim & 5 & 3 & 8 & 7 & 4 & 12 & 39 & 1,04 \\
\hline
\end{tabular}


Tapura_capitulifera

Theobroma_subincanum

Thyrsodium_guianense

Tovomita_sp.2_DS

Tovomita_sp.P4

Trymatococcus_oligandrus

Unonopsis_rufescens

Virola_michelii

Vouacapoua_americana

\begin{tabular}{lcccccccc} 
Tapcap & 19 & 63 & 2 & 30 & 45 & 134 & 293 & 7,81 \\
Thesub & 24 & 17 & 22 & 21 & 13 & 21 & 118 & 3,15 \\
Thygui & 15 & 14 & 7 & 1 & 8 & 22 & 67 & 1,79 \\
Tovsp2 & 16 & 19 & 24 & 13 & 25 & 26 & 123 & 3,28 \\
TovspP & 10 & 6 & 8 & 29 & 9 & 18 & 80 & 2,13 \\
Tryoli & 8 & 3 & 9 & 6 & 17 & 22 & 65 & 1,73 \\
Unoruf & 3 & 2 & 2 & 6 & 6 & 41 & 60 & 1,6 \\
Virmic & 10 & 7 & 24 & 4 & 4 & 2 & 51 & 1,36 \\
Vouame & 74 & 89 & 10 & 70 & 99 & 22 & 364 & 9,71 \\
\hline
\end{tabular}

